Article

\title{
Prevalence of Antimicrobial Resistant Bacteria from Conjunctival Flora in an Eye Infection Prone Breed (Saint Bernard)
}

\author{
George Cosmin Nadăș ${ }^{1,+} \mathbb{D}$, Cristiana Ștefania Novac ${ }^{1,+} \mathbb{D}$, Ioana Adriana Matei ${ }^{1, * \mathbb{D}}$, Cosmina Maria Bouari ${ }^{1}$, \\ Zoltan Miklos Gal ${ }^{2}$, Octavia Maria Tamas-Krumpe ${ }^{3}$, Adrian Maximilian Macri ${ }^{4}$ and Nicodim Iosif Fit ${ }^{1}$
}

Citation: Nadăș, G.C.; Novac, C.Ș. Matei, I.A.; Bouari, C.M.; Gal, Z.M.; Tamas-Krumpe, O.M.; Macri, A.M.; Fit, N.I. Prevalence of Antimicrobial Resistant Bacteria from Conjunctival Flora in an Eye Infection Prone Breed (Saint Bernard). Molecules 2021, 26, 2219. https://doi.org/10.3390/ molecules26082219

Academic Editors: Mihai

Cosmin Cenariu, Sanda Andrei and Adela Pintea

Received: 23 February 2021

Accepted: 9 April 2021

Published: 12 April 2021

Publisher's Note: MDPI stays neutral with regard to jurisdictional claims in published maps and institutional affiliations.

Copyright: (C) 2021 by the authors Licensee MDPI, Basel, Switzerland. This article is an open access article distributed under the terms and conditions of the Creative Commons Attribution (CC BY) license (https:// creativecommons.org/licenses/by/ $4.0 /)$
1 Department of Microbiology, Faculty of Veterinary Medicine, University of Agricultural Sciences and Veterinary Medicine, 400372 Cluj-Napoca, Romania; gnadas@usamvcluj.ro (G.C.N.); cristiana.novac@usamvcluj.ro (C.Ș.N.); cosmina.bouari@usamvcluj.ro (C.M.B.); nfit@usamvcluj.ro (N.I.F.)

2 Department of Reproduction, Obstetrics and Pathology of Reproduction, Faculty of Veterinary Medicine, University of Agricultural Sciences and Veterinary Medicine, 400372 Cluj-Napoca, Romania; zoltan.gal@usamvcluj.ro

3 Department of Physiology, Faculty of Veterinary Medicine, University of Agricultural Sciences and Veterinary Medicine, 400372 Cluj-Napoca, Romania; octavia.tamas@usamvcluj.ro

4 Department of Animal Nutrition, Faculty of Veterinary Medicine, University of Agricultural Sciences and Veterinary Medicine, 400372 Cluj-Napoca, Romania; adrian.macri@usamvcluj.ro

* Correspondence: ioana.matei@usamvcluj.ro; Tel.: +40-7-2809-6105

+ These authors contributed equally to this work.

Abstract: The conjunctival bacterial resident and opportunistic flora of dogs may represent a major source of dissemination of pathogens throughout the environment or to other animals and humans. Nevertheless, contamination with bacteria from external sources is common. In this context, the study of the antimicrobial resistance (AMR) pattern may represent an indicator of multidrug resistant (MDR) strains exchange. The present study was focused on a single predisposed breed-Saint Bernard. The evaluated animals were healthy, but about half had a history of ocular disease/treatment. The swabs collected from conjunctival sacs were evaluated by conventional microbiological cultivation and antimicrobial susceptibility testing (AST). The most prevalent Gram-positive was Staphylococcus spp.; regardless of the history, while Gram-negative was Pseudomonas spp.; exclusively from dogs with a history of ocular disease/treatment. Other identified genera were represented by Bacillus, Streptococcus, Trueperella, Aeromonas and Neisseria. The obtained results suggest a possible association between the presence of mixed flora and a history of ocular disease/treatment. A high AMR was generally observed (90\%) in all isolates, especially for kanamycin, doxycycline, chloramphenicol and penicillin. MDR was recorded in Staphylococcus spp. and Pseudomonas spp. This result together with a well-known zoonotic potential may suggest an exchange of these strains within animal human populations and the environment.

Keywords: AMR; MDR; ocular flora; Pseudomonas; Saint Bernard; Staphylococcus

\section{Introduction}

The conjunctival bacterial resident population is an important component of the local defense, and it helps to prevent colonization by the overgrowth of potentially pathogenic agents [1,2]. The predominant saprophytic organisms of the dog eye are Gram-positive bacteria, while Gram-negative bacteria presence is rather uncommon. The most common reported Gram-positive bacteria are the members of Staphylococcus spp.; Bacillus spp.; Streptococcus spp. and Trueperella spp. Gram-negative bacteria such as Escherichia coli, Pseudomonas spp.; Neisseria spp. and Klebsiella spp. are also frequently reported [3-7]. However, their saprophytic or opportunistic nature is not well-documented or stated. The structure of saprophytic flora may be slightly influenced by different internal factors (age, 
sex and breed) or external factors (season, geography and climate) [5,8]. However, some of these factors may not have a statistically significant influence [5].

The knowledge regarding normal ocular flora structure is of major importance, since the same organisms are the predominant agents recovered from ocular infection $[3,5]$. Opportunistic bacteria such as: Staphylococcus spp.; Streptococcus spp.; Pseudomonas spp.; E. coli, Trueperella spp. and Bacillus cereus may become potential pathogens if tissue damage occurs or if the organism's resistance to infection is decreased [9]. Withal, the destruction of the normal ocular flora by long-term use of topical antimicrobials can result in overgrowth of pathogenic bacteria, yeasts or fungi [9]. In addition, conformational abnormalities such as entropion or ectropion may contribute to secondary opportunistic infections [8]. The entropion may represent a predisposing factor, since it causes conjunctiva irritation or even trauma with ulceration of the cornea [10]. The ectropion results in high exposure of the conjunctiva, potentially able to cause conjunctivitis and keratitis [10] For instance, Wang et al. [5] evaluated the prevalence of positive bacterial culture on 13 dog breeds, showing a higher prevalence in Pug, Pekingese and Schnauzer. Despite the increased exposure of Pug's conjunctiva (exophthalmic breed), which may explain the high prevalence of this breed, the small number of tested animals was not sufficient to establish the presence of a statistically significant difference [5].

Alongside other breeds with genetic predilections for conformational abnormalities, the Saint Bernard is highly predisposed to both entropion and ectropion, having a socalled "diamond eye" [10,11]. However, to the best of our knowledge, there are no studies regarding the influence of these abnormalities on the resident or opportunistic flora or on Saint Bernard predisposition to eye infections.

Ocular disease associated with bacterial agents, either primarily or secondarily, represents a common problem in small animal practice, especially from the treatment point of view [8]. The most frequently used formulas are mixtures between anti-inflammatory agents with two or even three antibiotics that cover a large spectrum of agents. Commonly used formulas are combinations between neomycin, polymyxin $B$ and gramicidin (solution); or neomycin, polymyxin B and bacitracin (ointment), with efficacy on both the Gram-negative bacteria (neomycin and polymyxin B) and Gram-positive bacteria (bacitracin and, gramicidin) reviewed by reference [12]. Other frequently used antimicrobial agents are aminoglycosides: $3 \%$ tobramycin and $0.3 \%$ gentamicin, fluoroquinolones: $0.3 \%$ ofloxacin, $0.3 \%$ ciprofloxacin (solution and ointment), $0.5 \%$ levofloxacin, $0.5 \%$ moxifloxacin, $0.3 \%$ gatifloxacin and $0.6 \%$ besifloxacin and chloramphenicol ( $1 \%$ ointment) reviewed by reference [12]. Due to the long-term and high-frequency use of these agents, different resident or opportunistic bacteria were reported in the last years as being resistant to all previously mentioned drugs [13-16]. Nevertheless, the resistance of bacteria to certain antimicrobials not necessarily used in topic therapy may indicate either an influence of systemic therapy (used for treating other infections) or a circulation of resistant bacteria within the human-animal-environment interface. In this context, our aims were to evaluate: (i) the bacteria diversity and their frequency of in Saint Bernard eyes, (ii) the possible influence of individual and external factors on bacteria diversity, (iii) the antimicrobial resistance (AMR) to commonly used broad-spectrum drugs of isolated bacteria and (iv) to discuss the possible dissemination of resistant bacteria within the human-animal-environment interface.

\section{Results}

The population included in this study was represented by 28 females $(56 \%)$ and 22 males (44\%) aged one to eight years (mean age 4.2). A total number of 26 dogs (52\%) had clinical ocular infections and were treated with topical antibiotic (Mibazon ${ }^{\circledR}: 2.5-\mathrm{g}$ tetracycline, 1.2-g erythromycin, 1.2-g neomycin sulphate and 0.06-g prednisolone $/ 100 \mathrm{~g}$ ointment) in the last year. In 41 (82\%, CI 95\%: 68.56-91.42) cases, bacteria were isolated. The predominant identified flora was Gram-positive bacteria (100\%), Gram-negative only being isolated in eight cases (19.51\%, CI 95\%: 8.82-34.87) (Tables 1 and 2). In twenty 
cases (48.78\%, CI 95\%: 32.88-64.87), a pure culture was obtained. In the remaining cases, the associations of two $(n=16,39.02 \%$, CI 95\%: 24.2-55.5), three $(n=4,9.76 \%$ CI 95\%: 2.72-23.13) or four $(n=1,2.44 \%$, CI 95\%: 0.06-12.86) genera were observed (Table 2). The most frequent identified genus was Staphylococcus, followed by Micrococcus and Trueperella (Table 1). Among Gram-positive genera, Streptococcus and Bacillus were less frequently isolated. Gram-negative-identified genera were Pseudomonas, Aeromonas and Neisseria (Table 1).

Table 1. The prevalence of bacterial genera identified in ocular secretion in Saint Bernard dogs.

\begin{tabular}{cc}
\hline Pathogen/Genus & Frequency $(\mathbf{n} / \mathbf{\%})$ \\
\hline Staphylococcus & $38 / 92.68$ \\
Micrococcus & $7 / 17.07$ \\
Trueperella & $9 / 13.04$ \\
Streptococcus & $5 / 12.2$ \\
Pseudomonas & $4 / 9.76$ \\
Aeromonas & $2 / 4.88$ \\
Neisseria & $2 / 4.88$ \\
Bacillus & $2 / 2.90$ \\
\hline
\end{tabular}

Table 2. The bacterial associations identified in ocular secretion in Saint Bernard dogs.

\begin{tabular}{|c|c|}
\hline Bacteria Detected & Number of Cases \\
\hline Staphylococcus, Trueperella & 5 \\
\hline Staphylococcus, Micrococcus & 3 \\
\hline Staphylococcus, Streptococcus & 1 \\
\hline Staphylococcus, Trueperella, Micrococcus & 1 \\
\hline Staphylococcus, Trueperella, Bacillus & 1 \\
\hline Staphylococcus, Micrococcus, Streptococcus & 1 \\
\hline Streptococcus, Micrococcus & 1 \\
\hline Streptococcus, Bacillus & 1 \\
\hline Staphylococcus, Pseudomonas ${ }^{1}$ & 4 \\
\hline Staphylococcus, Aeromonas ${ }^{1}$ & 1 \\
\hline Staphylococcus, Neisseria ${ }^{1}$ & 1 \\
\hline Staphylococcus, Trueperella, Neisseria ${ }^{1}$ & 1 \\
\hline $\begin{array}{c}\text { Staphylococcus, Trueperella, Streptococcus, } \\
\text { Aeromonas } 1\end{array}$ & 1 \\
\hline
\end{tabular}

${ }^{1}$ Association between Gram-positive and -negative bacteria.

Age and sex seem to have no influence on the presence and prevalence of the different identified genera. Instead, the history of clinical infections/treatment seems to have an impact on the flora structure. The prevalence of Gram-negative flora was significantly higher from a statistical point of view in treated dogs compared to untreated dogs $(30.43 \%$ vs. $5.56 \%, p=0.04)$. Streptococcus, Neisseria and Pseudomonas genera were exclusively isolated from dogs with antibiotic treatment history ( $p=0.03,0.19$ and 0.06 , respectively). However, in the case of Pseudomonas and Neisseria, the difference was not statistically significant due to the small number of positive samples.

The efficient antibiotics were ciprofloxacin, marbofloxacin and ofloxacin with $97.5 \%$, $80.5 \%$ and $82.9 \%$, respectively, susceptible isolates, followed by gentamicin, amoxicillin and clavulanic acid (AMC) both with $78.1 \%$ and tobramycin with $75.6 \%$ susceptible isolates. A moderate frequency of susceptible isolates was observed for florfenicol with $68.3 \%$, cephalexin $63.4 \%$, neomycin $61 \%$ and tetracycline with $58.5 \%$. Less efficient can be considered doxycycline $34.2 \%$ and penicillin and kanamycin, both with $22 \%$, and chloramphenicol with $17.1 \%$ susceptible isolates. All isolates were resistant to bacitracin. A medium percent of the isolates was resistant to two-to-four antibiotics (to two: $9.8 \%$, three: $4.9 \%$ and four: $17.1 \%$ ). The resistance to several drugs was recorded in $24.4 \%$ of the isolates for five antibiotics, $4.9 \%$ for six and $7.3 \%$ for seven. Resistance for at least half of the 
tested antimicrobials were in $4.9 \%$ of isolates for eight antibiotics, $9.8 \%$ for nine, $2.4 \%$ for 10 and $11,4.9 \%$ for 12 and 13 and $2.4 \%$ for 15 antibiotics. The resistance to multiple drugs (more than seven) was recorded for only several genera: in 12 Staphylococcus spp. strains, four Pseudomonas spp. strains, three Micrococcus spp. and one Streptococcus spp. strain. Within Staphylococcus spp.; the majority of isolates were resistant to bacitracin, chloramphenicol, kanamycin, doxycycline and penicillin, while only a few isolates were resistant to ciprofloxacin, marbofloxacin and AMC (Table 3). Pseudomonas spp. was highly resistant, being susceptible to ciprofloxacin, marbofloxacin and ofloxacin (Table 3). A single Staphylococcus spp. strain showed resistance to all tested antibiotics.

Table 3. The frequency of resistant bacteria isolates in Saint Bernard eyes to the tested antimicrobials.

\begin{tabular}{|c|c|c|c|c|c|c|c|c|}
\hline & \multicolumn{8}{|c|}{ Bacterial Genera } \\
\hline & Staphylococcus & Streptococcus & Trueperella & Bacillus & Micrococcus & Aeromonas & Pseudomonas & Neisseria \\
\hline AMC & $6 / 38$ & $1 / 5$ & $0 / 9$ & $0 / 2$ & $1 / 7$ & $0 / 2$ & $4 / 4$ & $0 / 2$ \\
\hline $\mathrm{BAC}$ & $38 / 38$ & $5 / 5$ & $9 / 9$ & $2 / 2$ & $7 / 7$ & $2 / 2$ & $4 / 4$ & $2 / 2$ \\
\hline CAP & $33 / 38$ & $4 / 5$ & $7 / 9$ & $1 / 2$ & $5 / 7$ & $2 / 2$ & $4 / 4$ & $2 / 2$ \\
\hline CEX & $11 / 38$ & $2 / 5$ & $2 / 9$ & $0 / 2$ & $6 / 7$ & $0 / 2$ & $4 / 4$ & $0 / 2$ \\
\hline $\mathrm{CPF}$ & $1 / 38$ & $0 / 5$ & $0 / 9$ & $0 / 2$ & $0 / 7$ & $0 / 2$ & $0 / 4$ & $0 / 2$ \\
\hline DOX & $26 / 38$ & $3 / 5$ & $7 / 9$ & $1 / 2$ & $5 / 7$ & $2 / 2$ & $4 / 4$ & $2 / 2$ \\
\hline FLO & $13 / 38$ & $0 / 5$ & $1 / 9$ & $0 / 2$ & $2 / 7$ & $0 / 2$ & $4 / 4$ & $0 / 2$ \\
\hline GEN & $8 / 38$ & $1 / 5$ & $0 / 9$ & $0 / 2$ & $1 / 7$ & $0 / 2$ & $4 / 4$ & $0 / 2$ \\
\hline KAN & $31 / 38$ & $4 / 5$ & $8 / 9$ & $1 / 2$ & $4 / 7$ & $2 / 2$ & $3 / 4$ & $2 / 2$ \\
\hline MBX & $6 / 38$ & $0 / 5$ & $0 / 9$ & $0 / 2$ & $2 / 7$ & $0 / 2$ & $0 / 4$ & $0 / 2$ \\
\hline NEO & $15 / 38$ & $1 / 5$ & $1 / 9$ & $0 / 2$ & $2 / 7$ & $0 / 2$ & $4 / 4$ & $1 / 2$ \\
\hline OFX & $7 / 38$ & $0 / 5$ & $1 / 9$ & $0 / 2$ & $0 / 7$ & $0 / 2$ & $2 / 4$ & $0 / 2$ \\
\hline PCN & $28 / 38$ & $3 / 5$ & $6 / 9$ & $1 / 2$ & $6 / 7$ & $1 / 2$ & $4 / 4$ & $1 / 2$ \\
\hline TET & $15 / 38$ & $1 / 5$ & $1 / 9$ & $0 / 2$ & $3 / 7$ & $0 / 2$ & $4 / 4$ & $0 / 2$ \\
\hline TOB & $9 / 38$ & $1 / 5$ & $1 / 9$ & $0 / 2$ & $1 / 7$ & $0 / 2$ & $3 / 4$ & $0 / 2$ \\
\hline
\end{tabular}

Legend: AMC-Amoxicillin and clavulanic acid, BAC-Bacitracin, CAP-chloramphenicol, CEX-cephalexin, CPF-Ciprofloxacin, DOX—doxycycline, FLO—Florfenicol, GEN-Gentamicin, KAN—kanamycin, MBX-Marbofloxacin, NEO-Neomycin, OFX—Ofloxacin, $\mathrm{PCN}-$ penicillin, TET-Tetracycline and TOB-Tobramycin.

The ocular flora structure seems to be the only factor to have an influence on the frequency of resistant bacteria to multiple antimicrobials. The percent of Gram-negative bacteria resistant to more than seven antimicrobials was significantly higher, $p=0.01(100 \%$ Pseudomonas spp. vs. 60\% Micrococcus spp. or 31.6\% Staphylococcus spp.-resistant strains).

\section{Discussion}

Considering the common use of ophthalmic therapy in veterinary practice and the significance of AMR in the past decades, the study of ocular flora/pathogens and their AMR pattern is of major importance. In addition, the present study focused on one predisposed breed, highlighting the importance and influence of ocular disease and treatment history on normal ocular flora and the AMR pattern. Although the homogenous population with a common origin (same kennel) may be an advantage as the influencing external factors are similar, this aspect can be also a limitation of the study, since it is not clear how it will be applied to the general dog population.

In the present study, more than half of the Saint Bernard's included had ocular disease and treatment history. This is in agreement with the literature and well-known predisposition of this breed. This predisposition was associated with the length of the eyelid and the firmness of the lateral canthus, resulting in an overlong lid with little internal support that will naturally deform, while a slack lateral canthus allows the palpebral fissure to droop and distort [10]. According to the present observations, the Gram-positive flora, including Staphylococcus, Trueperella and Micrococcus genera, seem to predominate in both healthy and treated dogs, while Gram-negative microorganisms (i.e., Pseudomonas spp.; Aeromonas spp. and Neisseria) were more frequently detected in individuals with disease and treatment history. 
Despite the fact that members of the genus Staphylococcus are commonly isolated from ocular microbiota, being considered commensal on mucosal surfaces, many species may also act as opportunistic pathogens and cause eye-related infections [17-19]. Among different species of the genus, the most predominantly involved in ocular diseases seems to be S. intermedius, followed by S. aureus and less coagulase negative staphylococci [18]. Although the second-most detected genus in this study was Trueperella, other studies have suggested a minor involvement in the pathogenesis of ocular diseases [19,20]. Conversely, the genus Micrococcus seems to be detected from both normal and pathogenic flora [20-22].

Gram-negative rods are part of the normal microbiota but in a lower proportion $(\sim 10 \%)$ compared to Gram-positive ones $[20,23,24]$. However, the Gram-negative flora presence is important, since they usually may act as opportunistic bacteria. The most reported saprophytic flora is represented by the genera Moraxella, Acinetobacter and Neisseria [24,25]. In addition to these, Pseudomonas aeruginosa, Pasteurella spp.; Escherichia coli, Enterobacter spp.; Klebsiella spp. and Proteus spp. are also isolated from dogs with ocular disease history $[19,24,26]$. The difference in the frequency of Gram-negative rods (i.e., Pseudomonas spp.; Aeromonas spp. and Neisseria spp.) between the healthy and treated dogs observed in this study sustain the involvement of Gram-negative rods as opportunists $(p=0.04)$. Based on this, it can be assumed that prior treatment may help Gram-negative overgrowth. Although, in the present study, the prior treatment consisted of a triple combination (tetracycline, erythromycin and neomycin), the impact on Gram-negative overgrowth may be the same regardless of the antimicrobials used. In this case, Gram-negative bacteria (including resistant strains) that can act more often as opportunistic pathogens $[19,24,26]$ may circulate within the human-animal-environment interface, representing a public health risk.

The ocular components and, mainly, the conjunctive mucous membrane are constantly exposed to contamination with bacterial flora from the environment. These microbes may have developed a resistance to antibiotics, and, if they attach and colonize, ocular disease may develop. Furthermore, these antimicrobial-resistant microbes may be disseminated in the environment and to other susceptible hosts, including man. The dissemination of resistant bacteria within human-animal-environment interface has been extensively discussed and studied. White [27], in a review, discussed how the interaction between microecological systems in different hosts (including humans) and the environment may influence the transfer of resistant bacteria and their resistance genes [27]. Moreover, it highlights the pronounced capacity for the acquisition and transfer of resistance genes of commensals in ecosystems [27]. The transfer routes discussed in this review, by meat products or via other routes such as water and food plants [27], complement the direct transfer mechanism of zooanthroponotic agents within animal-human populations. Based on these considerations, the AMR pattern of the ocular flora may indicate a public health risk. In the present study, the most resistant isolates to antimicrobials were represented by Pseudomonas and Staphylococcus genera members.

The Pseudomonas genus includes P. aeruginosa, a well-known MDR zoonotic pathogen. According to the latest ECDC (European Center for Disease Prevention and Control) report, a high percentage of $P$. aeruginosa isolated from human blood cultures presented a resistance to several antimicrobial groups, including aminoglycosides, fluoroquinolones, carbapenems, etc. [28]. The zooanthroponotic potential of this species was previously reported $[29,30]$. In addition, the circulation of a resistant strain within pet owner-environment interfaces was also highlighted [29]. Unfortunately, in the veterinary sector, the surveillance of AMR is scarce. However, there are several reports on AMR P. aeruginosa isolated from domestic carnivores. For instance, Rubin et al. [31] isolated highly resistant $P$. aeruginosa strains from canine otitis and pyodermitis. A high percentage of resistance was recorded for $\beta$-lactams (ampicillin, cefoxitin, cefpodoxime, cephalothin, cefazolin, AMC and ceftiofur); for quinolones and fluoroquinolones (naladixic acid and orbifloxacin); to aminoglycosides (kanamycin and streptomycin) and to chloramphenicol and tetracycline [31]. Similarly, Li et al. [32] detected Pseudomonas spp. in clinical samples from 
domestic carnivores resistant to AMC, cefoxitin, ampicillin, cefotaxime and cefuroxime [32]. Pseudomonas aeruginosa strains isolated from ocular disease showed $100 \%$ susceptibility only to imipenem and a high resistance to enrofloxacin (75\%) [16]. All these observations are in agreement with the results reported here, showing a high resistance to most antimicrobial drugs ( $\beta$-lactams, aminoglycosides, cephalosporines, chloramphenicol and florfenicol), except for marbofloxacin. Overall, quinolones seem to have a better effectiveness against P. aeruginosa-specifically, marbofloxacin, norfloxacin, gatifloxacin, difloxacin, enrofloxacin, levofloxacin and ciprofloxacin $[16,31]$ in the present study.

The Staphylococcus genus includes species with zoonotic potential (e.g., S. aureus and S. pseudintermedius) and previously reported AMR. Although, according to the latest official reports of the ECDC, the MRSA (multidrug resistant Staphylococcus aureus) frequency is decreasing, it remains an important pathogen due to the combined resistance to other antimicrobial groups [28]. Both S. aureus and S. pseudintermedius (including methicillinresistant strains) have shown a zoonotic potential, being especially transmitted from dogs to humans [33-36]. Staphylococci, especially coagulase-negative species but, also, coagulasepositive ones (including S. aureus, S. intermedius and S. pseudintermedius) are commensals of the ocular surface with the potential to become opportunistic pathogens [18]. In the present study, staphylococci were isolated from both healthy dogs and dogs with ocular disease/treatment history. The majority of isolated strains presented are resistance to bacitracin, chloramphenicol, penicillin, kanamycin and doxycycline (Table 3). A variable frequency $(20-40 \%)$ of strains resistant to quinolones and $\beta$-lactams was recorded. In addition, 20 strains presented MDR. Similar results were obtained by Varges et al. [18], where a high resistance to penicillin and a moderate one to cephalexin and AMC was also observed for Staphylococcus spp. ocular isolates. Herein, a concerning percentage of MDR strains was reported [18]. Among different species of staphylococci, S. intermedius seems to have a limited resistance [13], whereas S. pseudintermedius seems to present a high resistance to different antimicrobial classes [15]. In this study, more than half of S. pseudintermedius strains were resistant to tetracycline, penicillin, sulfamethoxazole/trimethoprim, erythromycin and gentamycin [15]. Notably, a relatively high percentage (34\%) of these strains also presented resistance to oxacillin, suggesting a high prevalence of MRSP (multidrug resistant Staphylococcus pseudintermedius) [15]. Accordingly, MRS strains, including S. epidermidis, S. pseudintermedius and S. aureus, were isolated from canine corneal samples [37]. Although in the present study, the resistance to methicillin was not evaluated, the detection of MRS strains in other studies is of major importance, since the resistance to methicillin seems to be associated with a cross-resistance to other antimicrobial classes, as it was shown for some fluoroquinolones (ciprofloxacin and ofloxacin) [14].

The overall antimicrobial resistance patterns observed in the present study, although evaluated by the disk diffusion test, are comparable with the literature. This study included broad-spectrum antimicrobials used in both veterinary and human medicine, aiming to highlight the possible circulation of these bacteria within different hosts and the associated risk, altogether raising high concerns regarding the possible circulation of resistant strains in animals. Since most of detected bacteria have a zoonotic potential, they may also be transmitted to humans, either directly or through environmental contamination, representing a public threat.

\section{Materials and Methods}

\subsection{Animal and Specimen Collection}

A total of fifty Saint Bernard dogs from a private kennel with no clinical signs of conjunctivitis were evaluated during April-September 2018 regarding the presence, type of microbial flora and susceptibility to antimicrobial agents. The dogs were healthy at the beginning of the study, their health status confirmed by the owner and the result of a general examination.

The samples were collected using sterile cotton swabs, dry and premoistened in sterile saline. The sampling involved separate eye collection, and no anesthetics were applied. 
The swabs were used to swipe and roll the lower conjunctival sac directed to the nictitating membrane, avoid touching neighboring areas, such as eyelid margins, genes or cornea, that may contaminate the sample. Sterile Amies medium tubes for transportation were used for transfer. The samples were transported the same day to the microbiology laboratory.

\subsection{Bacteriological Examination}

In the microbiology laboratory, each swab was used to inoculate Columbia agar with 5\% sheep blood and MacConkey agar using the T-streak technique (three sections) and incubated under aerobic conditions at $36 \pm 2{ }^{\circ} \mathrm{C}$ for $18-24 \mathrm{~h}$. The swabs were then placed into a thioglycolate broth, used as a supplementary enrichment culture and again incubated under aerobic conditions at $36 \pm 2{ }^{\circ} \mathrm{C}$ for $18-24 \mathrm{~h}$. The next day, samples from the broth were used to inoculate Columbia agar with $5 \%$ sheep blood and incubated in aerobic conditions at $36 \pm 2{ }^{\circ} \mathrm{C}$ for $18-24 \mathrm{~h}$. The identification of bacteria at the genus level was performed by microscopy (Gram staining) cultural examination, followed by biochemical characters (Supplementary Table S1). Randomly selected strains (approx. 10\%) were identified using the Vitek ${ }^{\circledR} 2$ compact 15 system (bioMérieux, Marcy l'Etoile, France) confirming the genus level identification by the protocol used in this study (Supplementary Table S1).

\subsection{In Vitro Susceptibility Testing}

The evaluation of susceptibility to antimicrobials was performed using the disk diffusion method. Mueller-Hinton agar plates were flooded with $1 \mathrm{~mL}$ from the suspension of isolated colonies in broth at a $0.5 \mathrm{McF}$ arland density scale using Densicheck ${ }^{\mathrm{TM}}$ Plus (bioMérieux, Marcy l'Etoile, France). The antibiotics were represented by AMC 20/10 $\mu$, Bacitracin 0.04UI, chloramphenicol $30 \mu \mathrm{g}$, cephalexin $30 \mu \mathrm{g}$, ciprofloxacin $5 \mu \mathrm{g}$, doxycycline $30 \mu \mathrm{g}$, Florfenicol $30 \mu \mathrm{g}$, gentamicin $10 \mu \mathrm{g}$, kanamycin $30 \mu \mathrm{g}$, marbofloxacin $5 \mu \mathrm{g}$, neomycin $30 \mu \mathrm{g}$, ofloxacin $5 \mu \mathrm{g}$, penicillin 10UI, tetracycline $30 \mu \mathrm{g}$ and tobramycin $10 \mu \mathrm{g}$. Zone diameter breakpoints in mm were considered as follows: AMC 13, bacitracin 8, chloramphenicol 12, cephalexin 17 , ciprofloxacin 15 , doxycycline 18, florfenicol 18, gentamicin 12 , kanamycin 13, marbofloxacin 14, neomycin 14, ofloxacin 12, penicillin 24, tetracycline 17 and tobramycin 12, based on the lowest available EUCAST (European Committee on Antimicrobial Susceptibility Testing) and CLSI (Clinical and Laboratory Standards Institute) breakpoints for a certain genus (Supplementary Table S1).

\subsection{Statistical Analysis}

Statistical analysis was performed using Epi Info ${ }^{\mathrm{TM}} 7$ (CDC, Atlanta, GA, USA) software. The pathogen prevalence and their susceptibility to antibiotics and the prevalence of bacteria resistant to multiple antimicrobials $(>7)$ were analyzed with the chi-square independence test. Statistical significance was defined as $p<0.05$.

\section{Conclusions}

As it was observed in the present study and similar studies in the field, staphylococci represent major group of commensals that may act as opportunistic pathogens, being also frequently isolated from dogs with ocular disease history. Similarly, Gram-negative bacteria, including Pseudomonas spp.; are recognized as both commensals and opportunists. Herein, a higher frequency of mixed Gram-positive and -negative flora in dogs with ocular disease/treatment history were observed. Due to the small sample number included in the present study, it is not clear whether Gram-negative bacteria act more often as opportunistic or if the local treatment imbalanced the microflora structure; therefore, this aspect should be further investigated. Of major concern were the common AMR and MDR observed in both the most prevalent Gram-positive (i.e., Staphylococcus spp.) and Gram-negative (i.e., Pseudomonas spp.) members. The presence of MDR strains in dogs may represent an important threat from the One Health point of view, due to the likely dissemination of these strains within animal and human populations and the environment. 
Supplementary Materials: Table S1: Supplementary data of bacterial genera identification criteria and breakpoints establishing criteria.

Author Contributions: Conceptualization, N.I.F.; methodology, G.C.N.; C.Ș.N. and N.I.F.; software, I.A.M.; validation, N.I.F.; formal analysis, G.C.N.; C.S.N. and I.A.M.; investigation, G.C.N.; C.S.N. and Z.M.G.; resources, C.M.B.; A.M.M. and N.I.F.; writing-original draft preparation, G.C.N. and I.A.M.; writing-review and editing, C.S.N, O.M.T.-K. and N.I.F. and supervision, I.A.M. and N.I.F. All authors have read and agreed to the published version of the manuscript.

Funding: This research received no external funding.

Institutional Review Board Statement: This study was approved by the USAMV CN Bioethics committee with the registration number 23/21-09-2015, following the EU 2010/63 and National directives Ord. 28/31-08-2011 and National Law 206/2004. Permission to perform the study was also obtained from the kennel owner.

Informed Consent Statement: Not applicable.

Data Availability Statement: No new data were created or analyzed in this study. Data sharing is not applicable to this article.

Acknowledgments: We would like to thank to the kennel owner for accepting the collection of the samples and for the consent to submit this manuscript.

Conflicts of Interest: The authors declare no conflict of interest.

Sample Availability: Not available.

\section{References}

1. Miller, D.; Iovieno, A. The role of microbial flora on the ocular surface. Curr. Opin. Allergy Clin. Immunol. 2009, 9, 466-470. [CrossRef] [PubMed]

2. Willcox, M.D. Characterization of the normal microbiota of the ocular surface. Exp. Eye Res. 2013, 117, 99-105. [CrossRef]

3. Prado, M.R.; Rocha, M.F.; Brito, É.H.; Girão, M.D.; Monteiro, A.J.; Teixeira, M.F.; Sidrim, J.J. Survey of bacterial microorganisms in the conjunctival sac of clinically normal dogs and dogs with ulcerative keratitis in Fortaleza, Ceará, Brazil. Vet. Ophthalmol. 2005, 8, 33-37. [CrossRef] [PubMed]

4. Kudirkienè, E.; Žilinskas, H.; Šiugždaitè, J. Microbial flora of the dog eyes. Vet. Zootech. 2006, 34, $18-21$.

5. Wang, L.; Pan, Q.; Zhang, L.; Xue, Q.; Cui, J.; Qi, C. Investigation of bacterial microorganisms in the conjunctival sac of clinically normal dogs and dogs with ulcerative keratitis in Beijing, China. Vet. Ophthalmol. 2008, 11, 145-149. [CrossRef]

6. Garbacz, K.; Żarnowska, S.; Piechowicz, L.; Haras, K. Pathogenicity potential of Staphylococcus pseudintermedius strains isolated from canine carriers and from dogs with infection signs. Virulence 2013, 4, 255-259. [CrossRef] [PubMed]

7. Verneuil, M.; Durand, B.; Marcon, C.; Guillot, J. Conjunctival and cutaneous fungal flora in clinically normal dogs in southern France. J. Mycol. Med. 2014, 24, 25-28. [CrossRef]

8. Whitley, R.D. Canine and feline primary ocular bacterial infections. Vet. Clin. Small Anim. Pract. 2000, 30, 1151-1167. [CrossRef]

9. Gerding, P.A.; Kakoma, I. Microbiology of the canine and feline eye. Vet. Clin. N. Am. Small Anim. Pract. 1990, $20,615-625$. [CrossRef]

10. Bedford, P.G.C. Conditions of the eyelids in the dog. J. Small Anim. Pract. 1988, 29, 416-428. [CrossRef]

11. Miller, P.E. Breed Predisposition to Eye Disorders. In Slatter's Fundamentals of Veterinary Ophthalmology, 5th ed.; Maggs, D.J., Miller, P.E., Ofri, R., Eds.; Saunders Elsevier: St. Louis, MO, USA, 2013; p. 475.

12. Clode, A. Ophthalmic Pharmacology. In Veterinary Pharmacology and Therapeutics, 10th ed.; Riviere, E.J., Papich, M.G., Eds.; John Wiley Blackwell: Hoboken, NJ, USA, 2018; pp. 1326-1328.

13. Tolar, E.L.; Hendrix, D.V.; Rohrbach, B.W.; Plummer, C.E.; Brooks, D.E.; Gelatt, K.N. Evaluation of clinical characteristics and bacterial isolates in dogs with bacterial keratitis: 97 cases (1993-2003). J. Am. Vet. Med. 2006, 228, 80-85. [CrossRef]

14. D'Arienzo, P.A.; Wagner, R.S.; Jamison, T.; Bell, B.; Dajcs, J.J.; Stroman, D.W. Comparison of fluoroquinolone kinetics of kill in susceptible and resistant gram-positive conjunctival pathogens. Adv. Ther. 2010, 27, 39-47. [CrossRef]

15. Kang, M.H.; Chae, M.J.; Yoon, J.W.; Kim, S.G.; Lee, S.Y.; Yoo, J.H.; Park, H.M. Antibiotic resistance and molecular characterization of ophthalmic Staphylococcus pseudintermedius isolates from dogs. J. Vet. Sci. 2014, 15, 409-415. [CrossRef] [PubMed]

16. Leigue, L.; Montiani-Ferreira, F.; Moore, B.A. Antimicrobial susceptibility and minimal inhibitory concentration of Pseudomonas aeruginosa isolated from septic ocular surface disease in different animal species. Open Vet. J. 2016, 6, 215-222. [CrossRef] [PubMed]

17. Murphy, J.M.; Lavach, J.D.; Severin, G.A. Survey of conjunctival flora in dogs with clinical signs of external eye disease. J. Am. Vet. Med. Assoc. 1978, 172, 66-68. [PubMed]

18. Varges, R.; Penna, B.; Martins, G.; Martins, R.; Lilenbaum, W. Antimicrobial susceptibility of Staphylococci isolated from naturally occurring canine external ocular diseases. Vet. Ophthalmol. 2009, 12, 216-220. [CrossRef] [PubMed] 
19. Tuft, S.J.; Matheson, M. In vitro antibiotic resistance in bacterial keratitis in London. Br. J. Ophthalmol. 2000, 84, 687-691. [CrossRef] [PubMed]

20. Rogers, C.M.; Scott, E.M.; Sarawichitr, B.; Arnold, C.; Suchodolski, J.S. Evaluation of the bacterial ocular surface microbiome in ophthalmologically normal dogs prior to and following treatment with topical neomycin-polymyxin-bacitracin. PLoS ONE 2020, 15, e0234313. [CrossRef] [PubMed]

21. Malmasi, A.; SelkGhaffari, M.; Sadeghi-Hashin, G.; Davoodi, M.; Capiau, E.; Sharifian Fard, M.; Ahadinejad, S.; Bahonar, A. The effects of topical chloramphenicol and ciprofloxacin on conjunctival bacterial flora of healthy dogs. Iran J. Vet. Med. 2015, 9, 27-31. [CrossRef]

22. Sandmeyer, L.S.; Bauer, B.S.; Mohaghegh Poor, S.M.; Feng, C.X.; Chirino-Trejo, M. Alterations in conjunctival bacteria and antimicrobial susceptibility during topical administration of ofloxacin after cataract surgery in dogs. Am. J. Vet. Res. 2017, 78, 207-214. [CrossRef]

23. McDonald, P.J.; Watson, A.D.J. Microbial flora of normal canine conjunctivae. J. Small Anim. Pract. 1976, 17, 809-812. [CrossRef]

24. Svetikienè, D.; Grigonis, A.; Zamokas, G.; Karvelienè, B.; Babickaitè, L.; Ivaškienè, M.; Sugzdiniene, R. Conjunctival microbiota susceptibility to antibacterials in dogs. Vet. Zootech. 2020, 77, 38-44.

25. Banks, K.C.; Giuliano, E.A.; Reinero, C.R.; Ericsson, A.C. Evaluation of healthy canine conjunctival, periocular haired skin, and nasal microbiota compared to conjunctival culture. Front. Vet. Sci. 2020, 7, 558. [CrossRef]

26. Auten, C.R.; Urbanz, J.L.; Dees, D.D. Comparison of bacterial culture results collected via direct corneal ulcer vs. conjunctival fornix sampling in canine eyes with presumed bacterial ulcerative keratitis. Vet. Ophthalmol. 2020, 23, 135-140. [CrossRef] [PubMed]

27. Witte, W. Ecological impact of antibiotic use in animals on different complex microflora: Environment. Int. J. Antimicrob. Agents 2000, 14, 321-325. [CrossRef]

28. European Centre for Disease Prevention and Control. Available online: https://www.ecdc.europa.eu/en/publications-data/ surveillance-antimicrobial-resistance-europe-2019 (accessed on 15 February 2021).

29. Fernandes, M.R.; Sellera, F.P.; Moura, Q.; Carvalho, M.P.; Rosato, P.N.; Cerdeira, L.; Lincopan, N. Zooanthroponotic transmission of drug-resistant Pseudomonas aeruginosa, Brazil. Emerg. Infect. Dis. 2018, 24, 1160-1162. [CrossRef] [PubMed]

30. Mohan, K.; Fothergill, J.L.; Storrar, J.; Ledson, M.J.; Winstanley, C.; Walshaw, M.J. Transmission of Pseudomonas aeruginosa epidemic strain from a patient with cystic fibrosis to a pet cat. Thorax 2008, 63, 839-840. [CrossRef] [PubMed]

31. Rubin, J.; Walker, R.D.; Blickenstaff, K.; Bodeis-Jones, S.; Zhao, S. Antimicrobial resistance and genetic characterization of fluoroquinolone resistance of Pseudomonas aeruginosa isolated from canine infections. Vet. Microbiol. 2008, 131, 164-172. [CrossRef]

32. Li, Y.; Fernández, R.; Durán, I.; Molina-López, R.A.; Darwich, L. Antimicrobial Resistance in Bacteria Isolated from Cats and Dogs from the Iberian Peninsula. Front. Microbiol. 2021, 11, 3628. [CrossRef] [PubMed]

33. Van Duijkeren, E.; Wolfhagen, M.J.; Box, A.T.; Heck, M.E.; Wannet, W.J.; Fluit, A.C. Human-to-dog transmission of methicillinresistant Staphylococcus aureus. Emerg. Infect. Dis. 2004, 10, 2235. [CrossRef]

34. Boost, M.V.; O’Donoghue, M.M.; James, A. Prevalence of Staphylococcus aureus carriage among dogs and their owners. Epidemiol. Infect. 2008, 136, 953-964. [CrossRef] [PubMed]

35. Lozano, C.; Rezusta, A.; Ferrer, I.; Pérez-Laguna, V.; Zarazaga, M.; Ruiz-Ripa, L.; Revillo, M.J.; Torres, C. Staphylococcus pseudintermedius human infection cases in Spain: Dog-to-human transmission. Vector-Borne Zoonotic Dis. 2017, 17, 268-270. [CrossRef]

36. Somayaji, R.; Priyantha, M.A.R.; Rubin, J.E.; Church, D. Human infections due to Staphylococcus pseudintermedius, an emerging zoonosis of canine origin: Report of 24 cases. Diagn. Microbiol. Infect. Dis. 2016, 85, 471-476. [CrossRef] [PubMed]

37. LoPinto, A.J.; Mohammed, H.O.; Ledbetter, E.C. Prevalence and risk factors for isolation of methicillin-resistant Staphylococcus in dogs with keratitis. Vet. Ophthalmol. 2015, 18, 297-303. [CrossRef] [PubMed] 